This item was submitted to Loughborough's Research Repository by the author.

Items in Figshare are protected by copyright, with all rights reserved, unless otherwise indicated.

\title{
Knowledge is power? Outcome probability information impairs detection of deceptive intent
}

PLEASE CITE THE PUBLISHED VERSION

https://doi.org/10.1016/j.psychsport.2020.101744

PUBLISHER

Elsevier BV

VERSION

AM (Accepted Manuscript)

\section{PUBLISHER STATEMENT}

This paper was accepted for publication in the journal Psychology of Sport and Exercise and the definitive published version is available at https://doi.org/10.1016/j.psychsport.2020.101744.

LICENCE

CC BY-NC-ND 4.0

\section{REPOSITORY RECORD}

Jackson, Robin, Hayley Barton, and Daniel T Bishop. 2020. "Knowledge Is Power? Outcome Probability Information Impairs Detection of Deceptive Intent". Loughborough University.

https://hdl.handle.net/2134/12514283.v1. 


\section{Journal Pre-proof}

Knowledge is power? Outcome probability information impairs detection of deceptive intent

Robin C. Jackson, Hayley Barton, Daniel T. Bishop

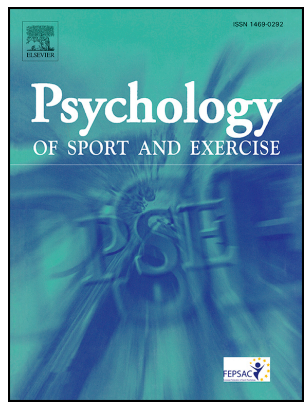

PII:

S1469-0292(20)30475-1

DOI:

https://doi.org/10.1016/j.psychsport.2020.101744

Reference: PSYSPO 101744

To appear in: Psychology of Sport \& Exercise

Received Date: 5 June 2020

Accepted Date: 5 June 2020

Please cite this article as: Jackson, R.C., Barton, H., Bishop, D.T., Knowledge is power? Outcome probability information impairs detection of deceptive intent, Psychology of Sport \& Exercise (2020), doi: https://doi.org/10.1016/j.psychsport.2020.101744.

This is a PDF file of an article that has undergone enhancements after acceptance, such as the addition of a cover page and metadata, and formatting for readability, but it is not yet the definitive version of record. This version will undergo additional copyediting, typesetting and review before it is published in its final form, but we are providing this version to give early visibility of the article. Please note that, during the production process, errors may be discovered which could affect the content, and all legal disclaimers that apply to the journal pertain.

(C) 2020 Published by Elsevier Ltd. 
Author contribution statement

Robin Jackson: Conceptualization, Methodology, Formal analysis, Data Curation, Writing Original draft, Supervision. Hayley Barton: Investigation, Resources, Data Curation, Writing - Review \& Editing, Project administration. Daniel Bishop: Resources, Writing - Review \& Editing, Supervision 
Knowledge is Power? Outcome Probability Information Impairs Detection of Deceptive Intent

\author{
Robin C. Jackson ${ }^{1}$
}

School of Sport, Exercise and Health Sciences, Loughborough University, UK

Hayley Barton

Division of Sport, Health and Exercise Sciences, Brunel University London, UK

Daniel T. Bishop

Division of Sport, Health and Exercise Sciences, Brunel University London, UK

${ }^{1}$ Corresponding author: Dr Robin Jackson

School of Sport, Exercise and Health Sciences

Loughborough University

Epinal Way

Loughborough

Leicestershire

LE11 3TU

Email: r.c.jackson@lboro.ac.uk

Author contribution statement

Robin Jackson: Conceptualization, Methodology, Formal analysis, Data Curation, Writing Original draft, Supervision. Hayley Barton: Investigation, Resources, Data Curation, Writing - Review \& Editing, Project administration. Daniel Bishop: Resources, Writing - Review \& Editing, Supervision 
Knowledge is Power? Outcome Probability Information Impairs Detection of Deceptive Intent

\begin{abstract}
The benefits and costs of prior expectations that are (i.e., congruent) or are not in harmony (i.e., incongruent) with action outcomes appear to be balanced; however, researchers have yet to examine the influence on skilled detection of deception. In this study we investigated whether response bias resulting from probability information (a) is stronger for low-skilled than high-skilled participants, (b) is stronger for deceptive actions than genuine actions, and (c) impairs the discriminability of genuine and deceptive actions. High-skilled $(n=15)$ and low-skilled $(\mathrm{n}=15)$ soccer players responded to life-sized projected video clips showing an oncoming opponent taking the ball to their left or right, with or without a deceptive 'stepover' action. Three probability conditions were used with respect to outcome direction: 50/50, $67 / 33$, and 83/17. Participants responded by stepping on one of two corresponding pressure mats, as if attempting to intercept the player. Response accuracy for genuine and deceptive actions was used to generate measures of bias $(c)$ and sensitivity $\left(d^{\prime}\right)$. The results confirmed stronger probability bias for deceptive actions than genuine ones, and for low-skilled than high-skilled participants. Congruence between high outcome probability and the direction of the fake significantly enhanced the effectiveness of the deceptive action. The study provides the first evidence that outcome probability information impairs skilled detection of deceptive intent.
\end{abstract}

Keywords: deception; anticipation; soccer

\title{
Introduction
}


Expert performers are highly attuned to opponent kinematics, which enables them to anticipate action outcomes better than their less-skilled counterparts (Mann, Williams, Ward, \& Janelle, 2007; Müller \& Abernethy, 2012). Researchers have shown that experts also use a variety of static and dynamic sources of contextual information to form accurate expectations about the most likely action outcome (Williams \& Jackson, 2019). These include the position of players relative to each other, the ball, and the court in racket sports (Abernethy, Gill, Parks, \& Packer, 2001; Murphy, Jackson, Cooke, Roca, Benguigui, \& Williams, 2016), the sequence of preceding actions and action outcomes (Farrow \& Reid, 2012; Gray, 2002; Loffing, Stern, \& Hagemann; 2015; Murphy, Jackson, \& Williams, 2019a), game situation and fielder positions in cricket (Runswick, Roca, Williams, McRobert, \& North, 2019), and individual player tendencies or action preferences (Gredin, Bishop, Broadbent, Tucker, \& Williams, 2018; Mann, Schaefers, \& Cañal-Bruland, 2014; Milazzo, Farrow, Ruffault, \& Fournier, 2016). However, few researchers have addressed the question of how contextual and kinematic sources of information combine to affect anticipation (Cañal-Bruland \& Mann, 2015; Loffing \& Cañal-Bruland, 2017). Moreover, while one study showed that head fakes were less effective when they were used with high relative frequency (Güldenpenning, Alaboud, Kunde, \& Weigelt, 2018) researchers have not examined how action outcome expectations affect skilled detection of deceptive intent (Güldenpenning, Kunde, \& Weigelt, 2017; Murphy, Jackson, \& Williams, 2019b). This is a crucial question because a body of research shows that the ability to detect deception is highly diagnostic of expertise, characterised by earlier differentiation of genuine and deceptive actions and better suppression of responses to deceptive signals (Brault, Bideau, Kulpa, \& Craig, 2012; Jackson, Warren, \& Abernethy, 2006; Jackson, Barton, Ashford, \& Abernethy, 2018). 
Accordingly, the purpose of the present study is to examine how action outcome probability information affects anticipation and detection of deception. We employed signal detection analysis to measure the extent to which outcome probability information biased participants' responses and affected their ability to discriminate between genuine and deceptive actions. Alongside its theoretical contribution, the practical significance of this study is underscored by the pervasiveness of deceptive actions in interactive duelling sports coupled with the widespread use of performance analysis to identify player preferences. For example, approximately half of all successful receptions in international netball matches were preceded by a deceptive action (Fox, Spittle, Otago, \& Saunders, 2014) and 72\% of successful tacklebreaks in rugby union were preceded by a sidestep (Wheeler, Askew, \& Sayers, 2010).

Situational probability and anticipation

The benefits of prior expectations that are congruent (i.e., in harmony) with action outcomes are well established. In a seminal study, Alain and Proteau (1977) recorded response times as participants struck one of two tennis balls suspended next to a light on their left and right. Before each block of trials, participants were told the probability that each light would illuminate, which ranged from 1.0/0 to 0.1/0.9. The researchers found that on congruent trials (i.e., when the higher-probability light illuminated) response times were faster for extreme values $(0.9 / 0.1 ; 0.1 / 0.9 ; 1.0 / 0)$ than in the equal probability condition $(0.5 / 0.5)$. Similar benefits of congruent high probability information were shown in studies that indirectly manipulated participant expectations. Skilled baseball batters made faster decisions, committed fewer errors, and timed their swings more accurately when contextual 'pitch count' information (e.g., balls, strikes, outs, runners on base) allowed them to identify the more likely pitch (Gray, 2002; Paull \& Glencross, 1997). Researchers have also shown that 
Joumal Pre-proof

higher-skilled performers are better at detecting statistical regularities. Tennis players progressively responded more quickly and accurately to the first serve of each block of eight when it was consistently directed to the same place, and karate players did so when an attack sequence was repeated every fourth trial (Farrow \& Reid, 2012; Milazzo, Farrow, Ruffault, \& Fournier, 2016).

A key question regarding congruence concerns the balance between the benefits and costs of congruent and incongruent action outcomes, respectively. Handball goalkeepers improved their ability to anticipate penalty throw direction but only when the thrower showed the same action preference as in training (Mann, Schaefers, \& Cañal-Bruland, 2014). Conversely, anticipation accuracy decreased when the thrower showed a different action preference in the post-test than in training. If the costs and benefits are balanced there is a mathematical advantage in knowing situational probabilities because, by definition, congruent action outcomes are more frequent. Evidence from studies of genuine actions supports this proposition. Relative to a 'no pre-cue' control condition, baseball batter response times were faster and response accuracy was slightly higher following a valid pre-cue, while response times were slightly slower and response accuracy was lower following an invalid pre-cue (Radlo, Janelle, Barba, \& Frehlich, 2001). Likewise, a simulated two-on-two soccer task revealed that high-skilled players with knowledge of the action preference of the player in possession of the ball to dribble $(67 \%)$ or pass to their teammate $(33 \%)$ were more accurate on congruent trials and less accurate on incongruent trials, relative to when they had no prior information (Gredin et al., 2018). A field-based study of soccer goalkeeper responses to penalty kicks similarly found equivalent costs and benefits for incongruent and congruent action outcomes (Navia, van der Kamp, \& Ruiz, 2013). On half of the trials, the ball was directed to the goalkeeper's left and right with equal probability. For the remaining trials there 
was a $0.8 / 0.2$ probability of the ball being kicked to each side of the goal. The goalkeepers dived to the correct side on approximately half of kicks across the equal probability and no probability conditions. The unequal probability condition led to an equivalent increase (highprobability side) and decrease (low-probability side) in the proportion of saves and dives to the correct side of the goal. Other studies in which there was no clear control condition nonetheless showed clear differences between congruent and incongruent action outcomes. In baseball, bat swing timing error for fast pitches was significantly greater when the pitch followed three slow pitches (incongruent sequence) than when it followed three fast pitches (congruent sequence, Gray, 2002). Similarly, skilled volleyball players responded more quickly and accurately when the outcome was congruent with the four preceding trials but were slower and less accurate when it was incongruent (Loffing et al., 2015).

Drawing on measures from signal detection theory (Swets, Tanner, \& Birdsall, 1961), researchers have argued that prior expectations will lead to cognitive strategies that bias responses on visual discrimination tasks but will not affect discriminability (Cañal-Bruland, Filius, \& Oudejans, 2015). In support, the researchers showed that baseball batters tended to 'sit on a fastball'; in other words, to bat as if expecting a fastball. Extending this account to perceptual judgment tasks that require different responses to left and right action outcomes, probability information would be expected to bias responses to genuine and deceptive actions equally, but have no effect on their discriminability. An alternative proposal, based on confirmation bias, is that prior expectations lead performers to place greater weight on kinematic information that confirms their expectation (Runswick et al., 2019). These researchers reasoned that skilled players would be more susceptible to performance decrements associated with incongruent action outcomes because they made greater use of contextual information. In support, skilled cricket batsmen were better than less-skilled 
batsmen at judging the outcome of deliveries (the point where the ball passed the stumps) but only when it was congruent with fielder positions, bowler type and game context. Conversely, high-skilled players performed worse than less-skilled players when outcome was incongruent with context.

Deceptive actions provide an opportunity to test these alternative accounts because attempts to 'sell the dummy' are often characterised by exaggeration in order to elicit an incorrect response. For example, foot displacement, head yaw and upper trunk yaw were found to be exaggerated in sidestep actions compared to actions without sidesteps (Brault, Bideau, Craig, \& Kulpa, 2010). In soccer stepovers, evidence of exaggeration was seen in participant responses to early-occluded video clips, which resulted in more incorrect responses than the equivalent correct responses to genuine actions. This led to a stronger reverse spatial cueing effect than the positive effect observed for genuine actions (Jackson et al., 2018; Wright \& Jackson, 2014). Spatial exaggeration increases the distinctiveness of images and actions, which in turn makes them easier to identify; for example, caricatures of famous faces tend to be recognised faster than veridical portraits (Mauro \& Kubovy, 1992). In regard to action recognition, researchers found that different styles of tennis serve were more accurately identified when their defining kinematic characteristics were exaggerated (Pollick, Fidopiastis \& Braden, 2001). Similarly, soccer goalkeepers anticipated the direction of non-deceptive penalty kicks more accurately when the kicker 'telegraphed' their intentions by exaggerating their kicking action (Smeeton \& Williams, 2012). Consistent with this property, we hypothesise that situational probability information will more strongly affect responses to deceptive actions than genuine actions, thereby increasing susceptibility to deception when the fake movement is aligned with outcome expectations. If correct, strong expectations will result in a greater increase in 'false alarm' responses to deceptive actions than correct 
responses to genuine actions. This, in turn, will impair the discriminability of genuine and deceptive actions.

To test this prediction, we used signal detection measures derived from the proportion of correct responses to one type of stimulus (i.e., genuine actions) and the corresponding proportion of 'false alarm' responses to the other type of stimulus (i.e., deceptive actions) in order to measure the discriminability of genuine and deceptive actions $\left(d^{\prime}\right)$ and participant bias in favour of judging an action to be genuine or deceptive $(c)$. These indices take into account the possibility that participants might have different levels of bias toward judging an action to be deceptive. To illustrate with an extreme example, an individual might achieve $100 \%$ accuracy when judging deceptive actions. However, until we know their accuracy on genuine trials we don't know to what extent this score represents perfect discrimination between genuine and deceptive actions (they score $100 \%$ on both: high discriminability, zero bias) or an extreme bias toward judging every action to be deceptive (they score $0 \%$ on genuine trials: high bias, zero discriminability). In other domains, signal detection analysis has been used to examine deception in facial expressions and personal injury narratives (Porter, Juodis, ten Brinke, Klein, \& Wilson, 2010), judgments of truth and deception in law enforcement (Meissner \& Kassin, 2002), and in comparisons of methods of eye-witness identification (Wixted \& Mickes, 2014). In sport research, signal detection analysis revealed that skilled handball goalkeepers and outfield players were equally good at differentiating genuine and deceptive penalty throws but that the goalkeepers showed a stronger bias toward judging throws to be deceptive (Cañal-Bruland \& Schmidt, 2009). Conversely, high-skilled soccer players were much better than low-skilled players at differentiating genuine and deceptive actions but both groups showed a bias to judge actions to be genuine (Jackson et al., 2018; Wright, Bishop, Jackson, \& Abernethy, 2013). 
In the present study we sought to test four specific hypotheses regarding the effect of outcome probability information on dynamic judgments of deceptive intent. Our first hypothesis $(\mathrm{H} 1)$ is that high-skilled soccer players will be better than low-skilled players at discriminating between genuine and deceptive actions. Our second hypothesis $(\mathrm{H} 2)$ is that probability information will more strongly bias responses of low-skilled performers than high-skilled performers, due to their relative inability to differentiate genuine and deceptive actions. In line with evidence for stronger responses to exaggerated actions, our third hypothesis $(\mathrm{H} 3)$ is that probability information will more strongly bias responses to deceptive actions than genuine actions. As a result of this imbalance, our fourth hypothesis $(\mathrm{H} 4)$ is that probability information will impair the discriminability of genuine and deceptive actions relative to the equal probability condition.

To test the first two hypotheses, we defined 'hits' as correct responses to genuine actions and 'false alarms' as incorrect responses to deceptive actions (Jackson et al., 2018). To test H3 and H4, we defined 'hits' as correct responses when the player took the ball to the high probability (congruent) side and 'false alarms' as incorrect responses when the player took the ball to the low-probability (incongruent) side. This second analysis must be conducted separately for genuine and deceptive actions and in this case response bias measures the tendency to respond in line with the higher probability value (negative $c$ values) or lower probability value (positive $c$ values). These values are then compared against the equal probability condition in which, by definition, there is no bias. The index of discriminability (d') measures the ability to discriminate between left and right action outcomes in each probability condition. 


\section{Method}

Participants. A medium effect size $(f=0.25)$ with Power set at 0.8 for two groups and five levels of the repeated measure yielded a recommended total sample size of 22 for the withinfactors and within-between interaction, and 28 for the tests involving three levels of the repeated measure. A total of 30 male soccer players were recruited. High-skilled participants $(n=15)$ were semi-professional players with a mean age of 20.6 years $(S D=2.4)$ and a mean of 14.2 years $(S D=2.8)$ of competitive playing experience. The low-skilled participants $(n=$ $15)$ had a mean age of 24.4 years $(S D=4.1$ years) and a mean of 1.3 years $(S D=1.2)$ of competitive playing experience at recreational level (e.g., local Sunday league club). Institutional ethical approval was granted and all participants gave written consent prior to participating in the study.

Test construction. We used a video camera (Canon HV40, Tokyo, Japan, 50 frames per second) mounted on a tripod at a height of $1.5 \mathrm{~m}$ to record high-definition sequences of two male right-footed players (hereafter 'actors') from an English Premier League academy. Each actor ran with the ball directly towards the video camera from a distance of $11.5 \mathrm{~m}$. They then changed direction to the left or right as they would when trying to evade another player. For non-deceptive actions, the actors simply manoeuvred the ball to the left or right of the camera with their lead foot. For the deceptive actions, the actors executed a 'stepover' by moving their lead foot in front of and across the ball before taking it in the opposite direction. The actors were instructed to perform as they would in a real one-on-one situation, running at pace directly towards the camera while maintaining close control of the ball. A panel of two UEFA B-license and one UEFA A-license coaches rated each video clip for the player's smoothness of movement, pace of the approach, and execution of the direction change. Overall mean 
scores were used to identify the 12 best examples from each player, which were used to develop the test stimuli.

The test comprised 12 blocks of 12 trials, containing six blocks from each actor. Actor order was counterbalanced across participants. The test trials for each actor comprised two blocks of 12 trials in each of the three levels of situational probability information $(50 / 50,67 / 33$, and 83/17); these values indicated the percentage likelihood that the actor would change direction to the left or right, respectively. For the $67 / 33$ and $83 / 17$ conditions, the higher probability value was associated with leftward direction changes for one block and rightward changes for the other. The order of presentation of the three levels of probability was counterbalanced across participants and was identical for the two actors. Each trial was occluded $80 \mathrm{~ms}$ after the lead foot contacted (genuine trials) or would have contacted (deceptive trials) the ball and was followed by a 5-second inter-trial interval. The test stimuli were presented using E-Prime software and were projected (Optoma HD25, CA, USA) to create a $1.6 \mathrm{~m} \mathrm{(h)} \mathrm{x} 2.1 \mathrm{~m}(\mathrm{w})$ scene. Participants stood $2.8 \mathrm{~m}$ from the screen and responded by stepping on one of two response mats ( $0.72 \times 0.39$ m, Defender Security, Farnell, Leeds, UK) placed on the floor in front, and to the left and right, of the participant.

Procedure. Participants were told they would view brief video clips designed to replicate a one-on-one scenario in which a player in possession runs towards them then takes the ball to their left or right. They were told that the actor in the video would simply take the ball to their left or right on half of the trials, and on the remaining trials would fake taking the ball one way before going the other by executing a 'stepover'. Participants were told that their task was to judge whether the actor would take the ball to their left or right and that they should respond by stepping on the left or right response pad. They were instructed to respond as 
quickly and accurately as possible as they would in an actual one-on-one situation.

Participants were informed that they would view 12 blocks of 12 trials and that the chance of the actor taking the ball to the left or right would be indicated on the screen before each block and would be either $50 / 50,67 / 33$ (or $33 / 67$ ), or $83 / 17$ (or 17/83). To familiarise participants with the experiment setup they were shown 16 trials, comprising four genuine and four deceptive trials from each actor. These video clips were different from those used in the test and probability information was not provided. During the test, the chance of the actor taking the ball to the left and right was displayed on the left and right side of the screen for 13 seconds before each block of 12 trials. Participants were given a 20 -second break after each block of trials and were given a two-minute break between the blocks of trials for the first actor (Blocks 1-6) and the second actor (Blocks 7-12).

Data analysis. Our first analysis focused on the extent to which different probability values (1) affected the ability to discriminate between genuine and deceptive actions, and (2) biased participants to judge actions to be genuine or deceptive. For this analysis, the index of discriminability $(d$ ') was calculated by subtracting $z$-scores for the proportion of incorrect responses on deceptive trials ('false alarms') from $z$-scores for the proportion of correct responses on genuine trials ('hits'). More positive values indicate better discrimination between genuine and deceptive actions. The criterion for responding $(c)$ was calculated by taking the average of the $z$-scores for 'hits' and 'false alarms'. Negative values indicate a bias to judge actions to be genuine and positive values indicated a bias to judge actions to be deceptive. The $d^{\prime}$ and $c$ indices were calculated for each of the five outcome probability values associated with the initial directional intention (left or right) conveyed by the action $(0.17,0.33,0.50,0.67,0.83)$. 
The second analysis focused on (1) the effect of each probability pair $(50 / 50,67 / 33$, and 83/17) on the discriminability of left and right action outcomes, and (2) the extent to which participant responses were biased in favour of the higher (or lower) probability value. For this analysis, the index of discriminability $\left(d^{\prime}\right)$ was calculated by subtracting $z$-scores for the proportion of incorrect responses on low probability outcomes ('false alarms') from $z$-scores for the proportion of correct responses on high probability outcomes ('hits'). The criterion for responding $(c)$ was calculated by taking the average of the $z$-scores for 'hits' and 'false alarms'.

To prevent infinite $z$-scores that result from scores of 0 and 1 , these values were replaced with 0.021 and 0.979 , respectively, using the formulae $1 / 2 \mathrm{n}$ and $(\mathrm{n}-0.5) / \mathrm{n}$ (Stanislaw \& Todorov, 1999), where $\mathrm{n}$ represents the number of normal and deceptive trials in the three probability conditions. The maximum value of $d^{\prime}$ was \pm 4.07 and for $c$ was \pm 2.03 . Alpha was set at .05 for all analyses and partial eta squared $\left(\eta_{\mathrm{p}}{ }^{2}\right)$ was used to indicate effect size. The GreenhouseGeisser adjustment to the degrees of freedom was applied when Mauchly's test of sphericity was violated.

Last, we recorded response time to examine a potential trade-off between response time and response accuracy. For each analysis the assumptions relating to parametric analyses and the $F$ distribution, such as normality, homogeneity of variances, independence of raw scores, and sphericity of the repeated measures values were evaluated. The univariate output was assessed with alpha set at .05 and the Greenhouse-Geisser correction was applied to the degrees of freedom when the sphericity assumption was violated. 


\section{Results}

The proportion of correct responses to genuine and deceptive actions for each of the five outcome probability values is shown in Figure 1, which has four features of interest. First, the high-skilled group (HS; square symbols) was much more accurate overall than the low-skilled group (LS; triangle symbols). Second, the difference in response accuracy for genuine trials (solid lines) and deceptive trials (dotted lines) was much greater in the LS group than the HS group. Third, as the outcome probability value associated with the initial direction change increased so too did the proportion of correct responses to genuine trials, whereas the proportion of correct responses to deceptive trials decreased. Fourth, the effect of the probability information appeared to be stronger for the LS group than the HS group.

\footnotetext{
**Figure 1 about here**
}

\section{Effect of outcome probability on the discriminability of genuine and deceptive actions}

The response sensitivity data across the five action outcome probabilities are shown in Figure 2. The HS group was substantially better than the LS group at discriminating between genuine and deceptive actions; that is, these participants made more 'hits' (correct responses on genuine trials) coupled with fewer 'false alarm' responses (incorrect responses on deceptive trials). Analysis of variance confirmed a significant and large effect of expertise, $F(1,28)=$ $54.76, p<.001, \eta_{\mathrm{p}}{ }^{2}=.66$, and a significant effect of outcome probability, $F(2.79,78.19)=$ $6.25, p=.001, \eta_{\mathrm{p}}{ }^{2}=.18$. High probability values resulted in a greater increase in 'false alarm' responses on deceptive trials than 'hits' on genuine trials (Figure 1). As a result, there was a notable decrease in the discriminability of genuine and deceptive actions for high probability 
values relative to the $50 \%$ condition. This was reflected in the results of simple contrast tests, which showed that sensitivity was significantly impaired in the $83 \%$ condition, $F(1,28)=$ $10.32, p=.003$, and not in the $17 \%(p=.95), 33 \%(p=.13)$, or $67 \%(p=.10)$ conditions. High-skilled players had a consistent advantage over less-skilled players across all five probability values, which was borne out by a non-significant Expertise x Probability interaction, $F(2.79,78.19)=0.40, p=.74, \eta_{\mathrm{p}}^{2}=.01$.

The response bias data (c) for the HS and LS groups are shown in Figure 3. We found strong support for the hypothesised relationship between outcome probability value and the degree of bias toward judging an action to be genuine, $F(2.39,66.85)=30.46, p<.001, \eta_{\mathrm{p}}{ }^{2}=.52$. Moreover, the significant and large effect of expertise showed that the probability information had a stronger effect on the the LS group than the HS group, $F(1,28)=20.66, p<.001, \eta_{\mathrm{p}}{ }^{2}=$ .43. The different slopes yielded a significant Expertise x Probability interaction, $F(2.39$, $66.85)=3.22, p=.04, \eta_{\mathrm{p}}{ }^{2}=.10$. One-tailed independent $t$-tests showed that bias toward judging actions to be genuine was significantly stronger in the LS group than the HS group for the $33 \%(p=.04,95 \% \mathrm{CI}=-0.02$ to 0.44$), 50 \%(p=.001,95 \% \mathrm{CI}=0.11$ to 0.42$), 67 \%(p$ $<.001,95 \% \mathrm{CI}=0.26$ to 0.77$)$, and $83 \%(p=.002,95 \% \mathrm{CI}=0.19$ to 0.95$)$ probability values.

\footnotetext{
**Figure 2 about here**

**Figure 3 about here**
}

\section{Bias toward responding to the high-probability side}

The degree to which probability information biased participant responses to genuine and deceptive actions is shown in Figure 4, in which values of zero for the 50/50 condition are included for reference. It is clear that the probability information biased participants to 
respond to the high-probability side and this bias increased from the $67 / 33$ to the $83 / 17$ condition. This was reflected in a significant effect of probability condition, $F(1,28)=9.91, p$ $=.004, \eta_{\mathrm{p}}{ }^{2}=.26$. Response bias was clearly stronger for deceptive actions than genuine actions in both groups and this was reflected in a significant effect of deception, $F(1,28)=$ 9.94, $p=.004, \eta_{\mathrm{p}}{ }^{2}=.26$. Consistent with analysis of the individual outcome probability values, bias to respond to the high-probability side was stronger in the LS group than the HS group, $F(1,28)=5.06, p=.03, \eta_{\mathrm{p}}^{2}=.15$. All other effects were non-significant.

The effect of probability information on task performance in genuine and deceptive trials is illustrated in Figure 5. Consistent with the data shown in Figure 1, the HS group was substantially better than the LS group at discriminating between left and right action outcomes, $F(1,28)=53.88, p<.001, \eta_{\mathrm{p}}{ }^{2}=.66$. The significant and large effect of deception confirmed that participants were much better at judging genuine actions than deceptive ones, $F(1,28)=87.13, p<.001, \eta_{\mathrm{p}}{ }^{2}=.66$. There was also a significant Expertise $\mathrm{x}$ Deception interaction, $F(1,28)=21.03, p<.001, \eta_{\mathrm{p}}^{2}=.43$, which was caused by a stronger effect of expertise on judgments of deceptive actions (HS: $M=3.23$; LS: $M=0.77$; difference $=2.46$ ) than genuine actions (HS: $M=3.85$; LS: $M=2.58$; difference $=1.27$ ). Last, there was a significant effect of probability, $F(2,56)=4.41, p=.02, \eta_{\mathrm{p}}{ }^{2}=.14$, which was caused by significantly lower discriminability scores in the 83-17 condition $(M=2.41, S E=0.13)$ than in the 50-50 condition $(M=2.72, S E=0.16)$ and the 67-33 condition $(M=2.69, S E=0.15)$. Of particular note, poorer discriminability for deceptive actions in the 83-17 condition reflected a larger increase in 'false alarm' responses when fakes were directed to the highprobability side than the equivalent decrease in 'false alarm' responses for fakes to the lowprobability side (see Figure 1). 
In sum, the signal detection analyses clearly show that probability information biased participant responses, that the effect was stronger for low-skilled than high-skilled performers, and that it was stronger for deceptive actions than genuine actions. As a result, the ability to discriminate between genuine and deceptive actions declined as probability values increased.

$$
\begin{aligned}
& * * \text { Figure } 4 \text { about here } * * \\
& * * \text { Figure } 5 \text { about here** }
\end{aligned}
$$

\section{Response time}

Analysis of response times for genuine and deceptive trials across the five outcome probability values revealed that high-skilled participants $(M=1734 \mathrm{~ms}, S E=26 \mathrm{~ms})$ responded faster than low-skilled participants $(M=1810 \mathrm{~ms}, S E=26 \mathrm{~ms}), F(1,28)=4.32, p$ $=.047, \eta_{\mathrm{p}}{ }^{2}=.13$. This indicates there was no speed-accuracy trade-off: the high-skilled group was both faster and more accurate. The analysis also revealed a small but statistically significant Expertise x Probability interaction, $F(4,112)=2.48, p=.048, \eta_{\mathrm{p}}{ }^{2}=.08$. As can be seen in Figure 6, response times for the HS group were consistent across probability values whereas response times of the LS group were slightly slower for the lower probability values. There was no difference in response times for genuine actions $(M=1773 \mathrm{~ms}, S E=18 \mathrm{~ms})$ and deceptive actions $(M=1771 \mathrm{~ms}, S E=20 \mathrm{~ms}), F(1,28)=0.06, p=.81, \eta_{\mathrm{p}}{ }^{2}=.00$.

\section{Discussion}


Journal Pre-proof

In recent review papers researchers highlighted the rudimentary understanding of how situational probability information affects anticipation and perception of deceptive intent (Jackson \& Cañal-Bruland, 2019; Müller \& Abernethy, 2012; Murphy et al., 2019b). The purpose of the present study was to begin to address this by determining how outcome probability information affects judgments of deceptive intent. To do so we analysed the degree to which probability information biased high-skilled and less-skilled participants' responses, and its effect on their ability to differentiate genuine and deceptive actions. The evidence in support of our central hypotheses was compelling. First, high-skilled participants were better than low-skilled counterparts at discriminating between genuine and deceptive actions (Jackson et al., 2018; Wright \& Jackson, 2014). In line with our second hypothesis, response bias associated with situational probability information was stronger for low-skilled participants than high-skilled participants (see Figure 3). In both groups, probability information biased participant responses in a consistent manner, characterised by a linear relationship between the response criterion $(c)$ and the outcome probability value (Figure 3 ). In simple terms, when the actor shaped to take the ball to the left or right, the tendency of participants to judge the action to be genuine changed as a function of outcome probability information. In line with our third hypothesis, the same probability information biased responses to deceptive actions more strongly than genuine ones and this was true for both high-skilled and low-skilled participants (see Figure 4). In line with our fourth hypothesis, this impaired discriminability of genuine and deceptive actions relative to the neutral (50/50) condition (Figure 5).

In regard to expertise, researchers have shown that experts make more effective use of dynamic contextual information than less-skilled players (Gredin, et al., 2018). In the present study we sought to examine how different levels of fixed prior probability information 
affected high- and low-skilled performers' judgments of deceptive intent. The results clearly show that performance on the primary task influenced the degree of bias afforded by prior probability information. Low-skilled participants were worse than high-skilled participants at discriminating between genuine and deceptive actions; correspondingly, low-skilled participants were more strongly influenced by prior probability information. This finding is consistent with a recent study that showed situational probability information more strongly influenced judgments when kinematic information was ambiguous (Helm, Cañal-Bruland, Mann, Troje, \& Munzert, 2020). In this study, the researchers systematically manipulated handball throw kinematics by applying a morphing procedure to create seven levels of kinematic ambiguity from exaggerated genuine actions (thrower released the ball) to exaggerated disguised actions (thrower retained the ball). Their results showed a curvilinear relationship between the degree of kinematic ambiguity and the effect of action preference information, with the effect strongest when the kinematic information was a 50-50 morph of genuine and disguised actions.

The present study replicates elements of these findings and extends them in several respects. First, we manipulated situational probability in regard to directional outcome (left, right) rather than preference for using genuine and disguised actions, which was held constant at $50 \%$. Accordingly, our results extend the findings from action preference to action outcome probabilities, which are arguably more typical of the information provided in many direction judgment tasks in sport (Navia et al., 2013). Second, we used high-skilled and low-skilled performers rather than novices. This allowed us to compare the effects of action outcome probability information on performers who differed in their ability to distinguish between genuine and deceptive actions. Importantly, the evidence from comparisons between the two groups corroborates and extends the findings of Helm et al. (2020), in that it supports the 
integration of kinematic and situational probability information in high-skilled as well as lowskilled performers, and in that the effect of probability information was stronger in performers who were less able to differentiate genuine and deceptive actions. Third, we occluded videos after the critical time-point (i.e., after the point at which the foot contacted or would have contacted the ball) and instructed participants to make physical responses similar to those they would make when defending against a real player. This revealed perhaps the most striking finding, which was that high-skilled performers did extremely well on deceptive trials when the probability information was balanced (50/50 condition) but were nonetheless significantly impaired when strong expectations aligned with the (false) intention conveyed by a deceptive action (see Figure 1). Researchers have shown that a key characteristic of expert judgments of deceptive intent is their ability to suppress incorrect responses to deceptive actions (Brault et al., 2012). We found evidence of this in the 50/50 condition, yet the ability was significantly and progressively impaired as outcome probabilities associated with the direction of the fake strengthened. In a physical sense, the probability information changed the criterion for responding, resulting in fewer suppressed incorrect responses. To quantify this effect in practical terms, interpolation of the data in Figure 4 suggests that the response bias for genuine actions in the $83 / 17$ probability condition could be attained in deceptive actions with probabilities of just 61/39 (high-skilled group) and 65/35 (low-skilled group).

It is important to note that the potential dual upsides of increased accuracy on high probability genuine trials and low probability deceptive trials were smaller in the high-skilled group than the low-skilled group because their baseline performance (50/50 condition) was higher. To confirm that probability information more strongly biased responses for low-skilled performers and for deceptive actions we inspected the relative downsides of more 'false alarm' responses on high probability deceptive trials and fewer 'hits' on low probability 
genuine trials, neither of which were subject to potential ceiling effects (Figure 1). In support of the stronger effect of probability information on low-skilled performers, response accuracy relative to the 50/50 condition decreased more in the low-skilled group than the high-skilled group in both instances. Likewise, in support of a stronger effect of probability information on deceptive actions, the decrease in response accuracy on high probability deceptive trials was greater than the decrease in response accuracy on low probability genuine trials.

In regard to the question of whether prior probability information leads to a cognitive bias or affects the way individuals interact with the task, the present results clearly show that probability information changed the judgment criterion more strongly for deceptive actions than genuine actions. As a result, participants incurred greater costs by way of more 'false alarm' responses to deceptive actions. These outweighed the benefits of more correct responses to genuine actions, which impaired their ability to discriminate between genuine and deceptive actions. This provides further evidence that prior probability information and kinematic information are integrated as the action unfolds (Gray \& Cañal-Bruland, 2018; Helm, et al., 2020). More importantly, the way they did so was consistent with confirmation bias, because response bias was stronger for the exaggerated confirmatory signals associated with deceptive actions than for the non-exaggerated signals in genuine actions (Runswick et al., 2019). We add a caveat to this interpretation, in that we did not analyse the kinematics of the video stimuli used in the test. Exaggeration has been identified in other deceptive actions such as rugby sidesteps and deceptive soccer penalty kicks, and is considered integral to 'selling' the dummy. Nonetheless, systematic manipulation of the degree of exaggeration used to convey deceptive intent is needed to demonstrate unequivocally its role in strengthening biasing effects. From a signal detection perspective, we speculate there will be a point at which the exaggerated action becomes so different to the equivalent genuine action 
that action outcome can be readily identified so will be less effective in inducing confirmation bias. Establishing this threshold, and how it is affected by prior expectations, will further enhance our understanding of how kinematic and contextual sources of information are integrated.

\section{Practical Implications}

The present study has two key practical implications. First, we question the efficacy of providing 'player preference' information for action outcomes in skills in which deceptive actions are common. Our results show that the performance benefits when action outcomes were congruent with expectations were outweighed by the costs incurred when the performer faked to move in the expected direction. In considering whether or not to provide such information, it is instructive that the key finding - namely, deceptive actions became superdeceptive when intent was aligned with high expectations - held true for both high-skilled and low-skilled participants. High-skilled participants responded correctly to almost $95 \%$ of deceptive trials in the neutral (50/50) condition yet this decreased to $76 \%$ when the fake intent was aligned with strong outcome expectations (Figure 1, 83\% probability). The potential benefits of prior probability information were clearly greater for low-skilled performers because they performed at a lower level $(65 \%)$ in the 50/50 condition; however, this was offset by their greater susceptibility to deception. Indeed low-skilled participants responded correctly on just $35 \%$ of trials when deceptive intent was aligned with the strongest expectations (Figure 1).

Relatedly, a second practical implication is that performers should consider how they might exploit the expectations of their opponent. Researchers have shown that unexpected action 
outcomes can be highly successful (Runswick, et al., 2019). The present study shows that by aligning deceptive intent conveyed by their actions with the outcome expectations of their opponent a performer can dramatically increase the potency of their deceptive action. This 'higher order' level of conditional deception can be used to exploit situations in which context leads to strong expectations regarding likely actions. It suggests, for example, that when player and court or field positions lead to a strong expectation on the part of a defensive player, the attacking player will be more successful by first faking to do the expected rather than simply doing the unexpected.

\section{Conclusion}

The present findings offer compelling evidence for the influence of prior expectations on the perception of deceptive intent and lead to three main conclusions. First, the influence of situational probability information was evident in both high-skilled and low-skilled performers, which highlights the potent effect of prior expectations, particularly when they align with the intention conveyed by a fake action. Second, the stronger influence of situational probability information on deceptive actions than genuine actions supports its active integration during the task in a manner that is consistent with confirmation bias. Third, the stronger influence of situational probability information on low-skilled than high-skilled performers corroborates findings regarding the different weighting given to this information depending on the difficulty of the primary task. In sum, the results lead us to caution that situational probability information might harm anticipation in certain sports skills because deceptive actions become 'super-deceptive' when aligned with observer expectations.

\section{Funding}


This research did not receive any specific grant from funding agencies in the public, commercial, or not-for-profit sectors.

Competing interests

There are no competing interests to declare.

References

Abernethy, B., Gill, D. P., Parks, S. L., \& Packer, S. T. (2001). Expertise and the perception of kinematic and situational probability information. Perception, 30, 233-252. doi:10.1068/p2872

Alain, C., \& Proteau, L. (1977). Perception of objective probabilities in motor performance. In B. Kerr (Ed.), Human performance and behaviour. Banff, Alberta.

Brault, S., Bideau, B., Craig, C., \& Kulpa, R. (2010). Balancing deceit and disguise: How to successfully fool the defender in a 1 vs. 1 situation in rugby. Human Movement Science, 29, 412-425. doi:10.1016/j.humov.2009.12.004

Brault, S., Bideau, B., Kulpa, R., \& Craig, C. M. (2012). Detecting deception in movement: The case of the side-step in rugby. PLoS ONE, 7(6): e37494. doi:10.1371/journal.pone.0037494

Cañal-Bruland, R., Filius, M. A., \& Oudejans, R. R. D. (2015). Sitting on a fastball. Journal of Motor Behavior, 47, 267-270. doi:10.1080/00222895.2014.976167

Cañal-Bruland, R., \& Mann, D. L. (2015). Time to broaden the scope of research on anticipatory behavior: A case for the role of probabilistic information. Frontiers in Psychology, 6: 1518. doi:10.3389/fpsyg.2015.01518 
Cañal-Bruland, R., \& Schmidt, M. (2009). Response bias in judging deceptive movements. Acta Psychologica, 130, 235-240.

Farrow, D., \& Reid, M. (2012). The contribution of situational probability information to anticipatory skill. Journal of Science and Medicine in Sport, 15, 368-373. doi:10.1016/j.jsams.2011.12.007

Fox, A., Spittle, M., Otago, L., \& Saunders, N. (2014). Offensive agility techniques performed during international netball competition. International Journal of Sports Science \& Coaching, 9, 543-552.

Gray, R. (2002). "Markov at the bat": A model of cognitive processing in baseball batters. Psychological Science, 13, 542-547. doi:10.1111/1467-9280.00495

Gray, R., \& Cañal-Bruland, R. (2018). Integrating visual trajectory and probabilistic information in baseball batting. Psychology of Sport and Exercise, 36, 123-131. doi:10.1016/j.psychsport.2018.02.009

Gredin, N. V., Bishop, D. T., Broadbent, D. P., Tucker, A., \& Williams, A. M. (2018). Experts integrate explicit contextual priors and environmental information to improve anticipation efficiency. Journal of Experimental Psychology-Applied, 24, 509-520. doi:10.1037/xap0000174

Güldenpenning, I., Alhaj Ahmad Alaboud, M., Kunde, W., \& Weigelt, M. (2018). The impact of global and local context information on the processing of deceptive actions in game sports. German Journal of Exercise and Sport Research, 48, 366-375. doi: $10.1007 / \mathrm{s} 12662-018-0493-4$

Güldenpenning, I., Kunde, W., \& Weigelt, M. (2017). How to trick your opponent: A review article on deceptive actions in interactive sports. Frontiers in Psychology, 8: 12. doi:10.3389/fpsyg.2017.00917 
Helm, F., Cañal-Bruland, R., Mann, D., Troje, N., \& Munzert, J. (2020). Integrating situational probability and kinematic information when anticipating disguised movements. Psychology of Sport \& Expertise, 46: 101607.

Jackson, R. C. \& Cañal-Bruland, R. (2019). Deception in sport. In A. M. Williams \& R. C. Jackson (Eds.) Anticipation and decision making in sport (pp. 99-116). Abingdon, Oxon: Routledge.

Jackson, R. C., Barton, H., Ashford, K. J., \& Abernethy, B. (2018). Stepovers and signal detection: Response sensitivity and bias in the differentiation of genuine and deceptive football actions. Frontiers in Psychology, 9: 2043. doi:10.3389/fpsyg.2018.02043

Jackson, R. C., Warren, S., \& Abernethy, B. (2006). Anticipation skill and susceptibility to deceptive movement. Acta Psychologica, 123, 355-371.

Loffing, F., \& Cañal-Bruland, R. (2017). Anticipation in sport. Current Opinion in Psychology, 16, 6-11. doi:10.1016/j.copsyc.2017.03.008

Loffing, F., Stern, R., \& Hagemann, N. (2015). Pattern-induced expectation bias in visual anticipation of action outcomes. Acta Psychologica, 161, 45-53. doi:10.1016/j.actpsy.2015.08.007

Mann, D. L., Schaefers, T., \& Cañal-Bruland, R. (2014). Action preferences and the anticipation of action outcomes. Acta Psychologica, 152, 1-9. doi:10.1016/j.actpsy.2014.07.004

Mann, D. T. Y., Williams, A. M., Ward, P., \& Janelle, C. M. (2007). Perceptual-cognitive expertise in sport: A meta-analysis. Journal of Sport \& Exercise Psychology, 29, 457478.

Mauro, R., \& Kubovy, M. (1992). Caricature and face recognition. Memory \& Cognition, 20, 433-440. doi:10.3758/bf03210927 
Meissner, C. A., \& Kassin, S. M. (2002). "He's guilty!": Investigator bias in judgments of truth and deception. Law and Human Behavior, 26, 469-480. doi:10.1023/a:1020278620751

Milazzo, N., Farrow, D., Ruffault, A., \& Fournier, J. F. (2016). Do karate fighters use situational probability information to improve decision-making performance during onmat tasks? Journal of Sports Sciences, 34, 1547-1556. doi:10.1080/02640414.2015.1122824

Müller, S., \& Abernethy, B. (2012). Expert anticipatory skill in striking sports: A review and a model. Research Quarterly for Exercise and Sport, 83, 175-187.

Murphy, C. P., Jackson, R. C., Cooke, K., Roca, A., Benguigui, N., \& Williams, A. M. (2016). Contextual information and perceptual-cognitive expertise in a dynamic, temporally-constrained task. Journal of Experimental Psychology-Applied, 22, 455-470. doi:10.1037/xap0000094

Murphy, C. P., Jackson, R. C., \& Williams, A. M. (2019a). Informational constraints, option generation, and anticipation. Psychology of Sport and Exercise, 41, 54-62. doi:10.1016/j.psychsport.2018.11.012

Murphy, C. P., Jackson, R. C., \& Williams, A. M. (2019b). Contextual information and its role in expert anticipation. In A. M. Williams \& R. C. Jackson (Eds.) Anticipation and decision making in sport (pp. 43-58). Abingdon, Oxon: Routledge.

Navia, J. A., van der Kamp, J., \& Ruiz, L. M. (2013). On the use of situation and body information in goalkeeper actions during a soccer penalty kick. International Journal of Sport Psychology, 44, 234-251. doi:10.7352/ijsp2013.44.234

Paull, G., \& Glencross, D. (1997). Expert perception and decision making in baseball. International Journal of Sport Psychology, 28, 35-56. 
Pollick, F. E., Fidopiastis, C., \& Braden, V. (2001). Recognising the style of spatially exaggerated tennis serves. Perception, 30, 323-338. doi:10.1068/p3064

Porter, S., Juodis, M., ten Brinke, L. M., Klein, R., \& Wilson, K. (2010). Evaluation of the effectiveness of a brief deception detection training program. Journal of Forensic Psychiatry \& Psychology, 21, 66-76. doi:10.1080/14789940903174246

Radlo, S. J., Janelle, C. M., Barba, D. A., \& Frehlich, S. G. (2001). Perceptual decision making for baseball pitch recognition: Using P300 latency and amplitude to index attentional processing. Research Quarterly for Exercise and Sport, 72, 22-31.

Runswick, O. R., Roca, A., Williams, A. M., McRobert, A. P., \& North, J. S. (2019). Why do bad balls get wickets? The role of congruent and incongruent information in anticipation. Journal of Sports Sciences, 37, 537-543. doi:10.1080/02640414.2018.1514165

Smeeton, N. J., \& Williams, A. M. (2012). The role of movement exaggeration in the anticipation of deceptive soccer penalty kicks. British Journal of Psychology, 103, 539555. doi:10.1111/j.2044-8295.2011.02092.x

Stanislaw, H., \& Todorov, N. (1999). Calculation of signal detection theory measures. Behavior Research Methods Instruments \& Computers, 31, 137-149. doi:10.3758/bf03207704

Swets, J. A., Tanner, W. P., \& Birdsall, T. G. (1961). Decision-processes in perception. Psychological Review, 68, 301-340. doi:10.1037/0033-295x.68.5.301

Wheeler, K. W., Askew, C. D., \& Sayers, M. G. (2010). Effective attacking strategies in rugby union. European Journal of Sport Science, 10, 237-242. doi:10.1080/17461391.2010.482595

Williams, A. M., \& Jackson, R. C. (2019). Anticipation in sport: Fifty years on, what have we learned and what research still needs to be undertaken? Psychology of Sport and Exercise, 42, 16-24. doi:10.1016/j.psychsport.2018.11.014 
Wixted, J. T., \& Mickes, L. (2014). A signal-detection-based diagnostic-feature-detection model of eyewitness identification. Psychological Review, 121, 262-276. doi:10.1037/a0035940

Wright, M., Bishop, D. T., Jackson, R. C., \& Abernethy, B. (2013). Brain regions concerned with the identification of deceptive soccer moves by higher-skilled and lower-skilled players. Frontiers in Human Neuroscience, 7:851.

Wright, M. J., \& Jackson, R. C. (2014). Deceptive body movements reverse spatial cueing in soccer. PLoS ONE, 9: 9. doi:10.1371/journal.pone.0104290 


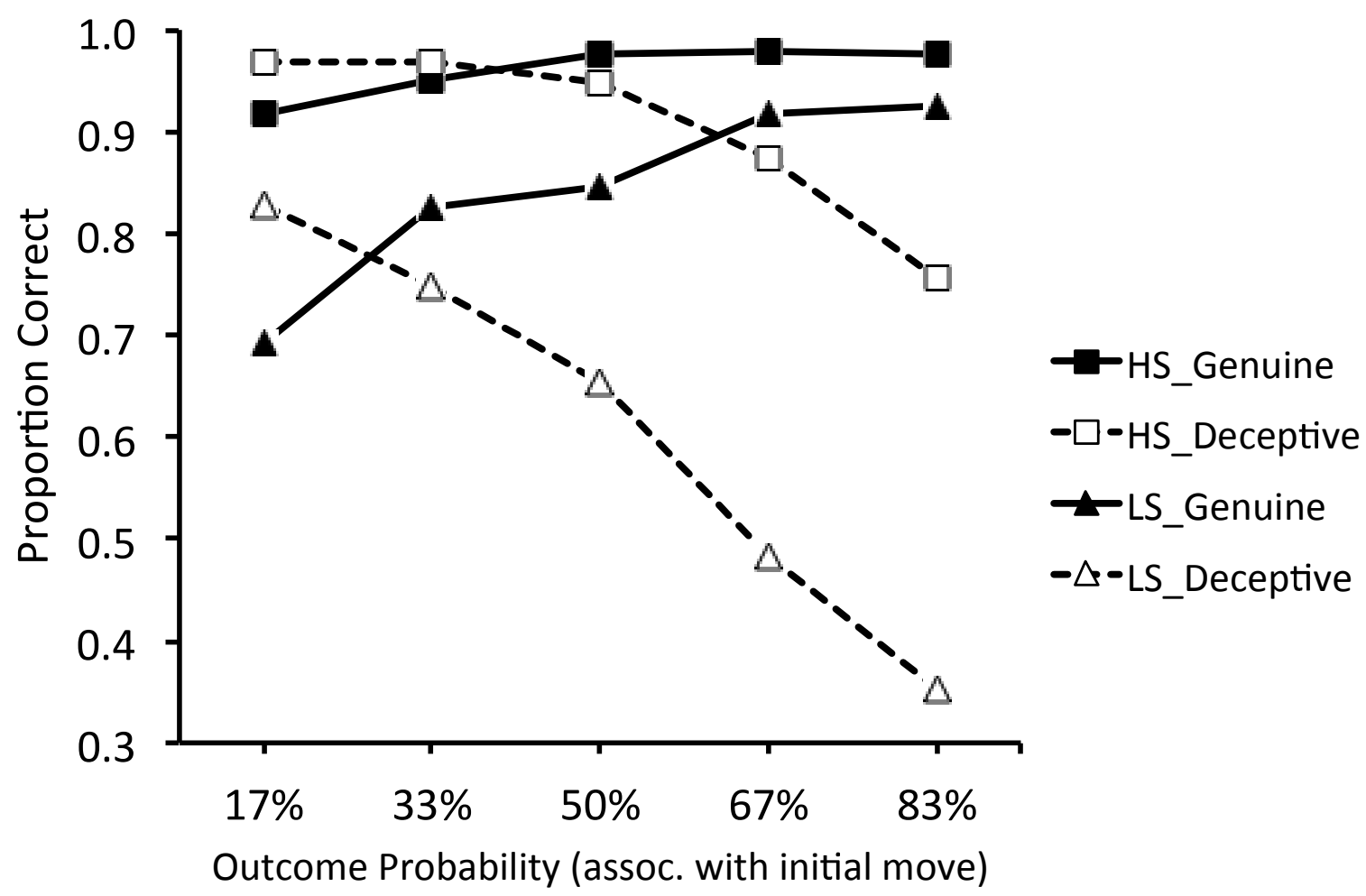

Figure 1. The proportion of correct responses made by the high-skilled (HS) and low-skilled (LS) group on genuine (solid lines) and deceptive (dashed lines) trials for each outcome probability value. 


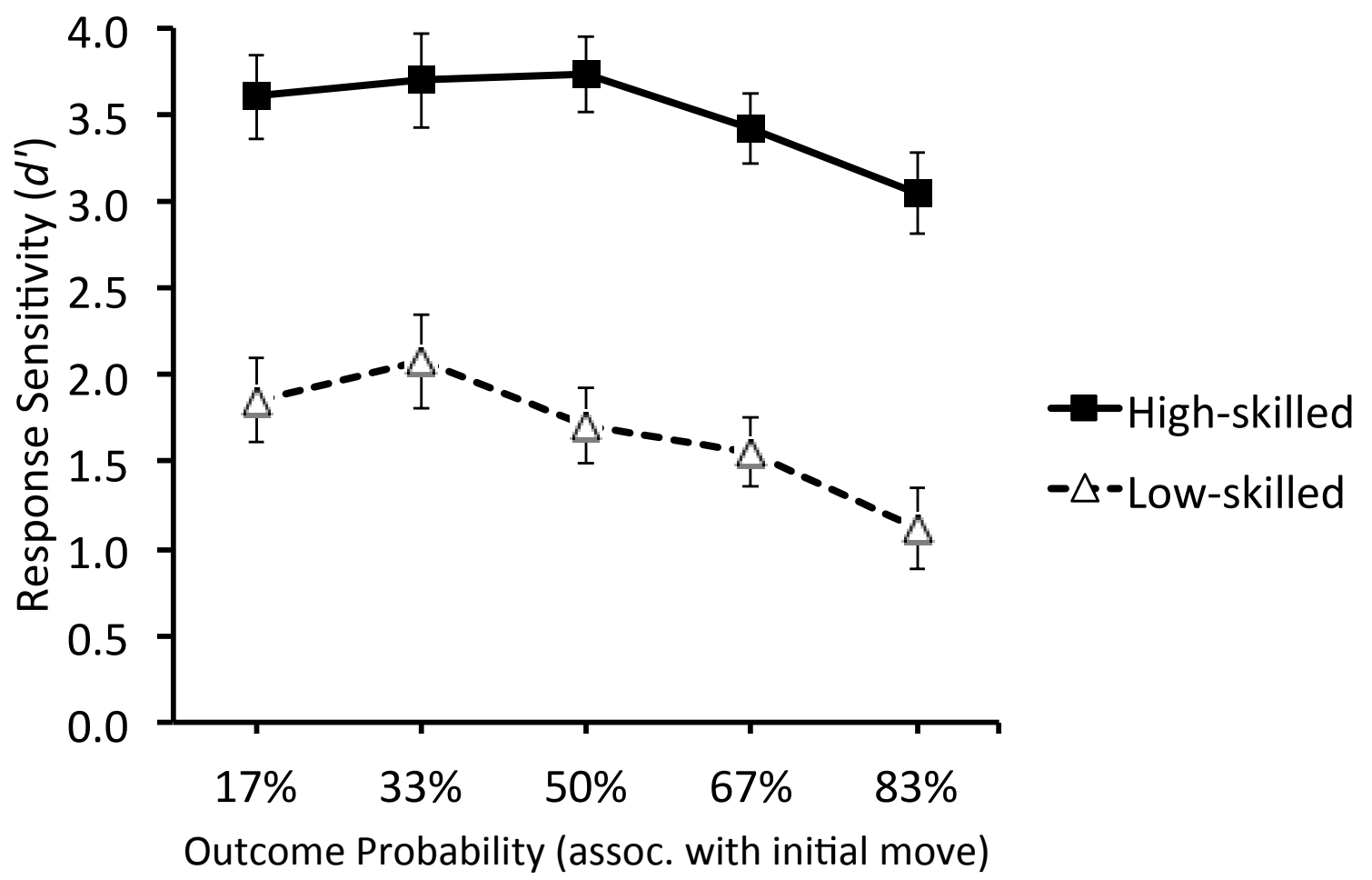

Figure 2. Sensitivity in discriminating between genuine and deceptive actions for the highskilled and low-skilled group for each outcome probability value. 


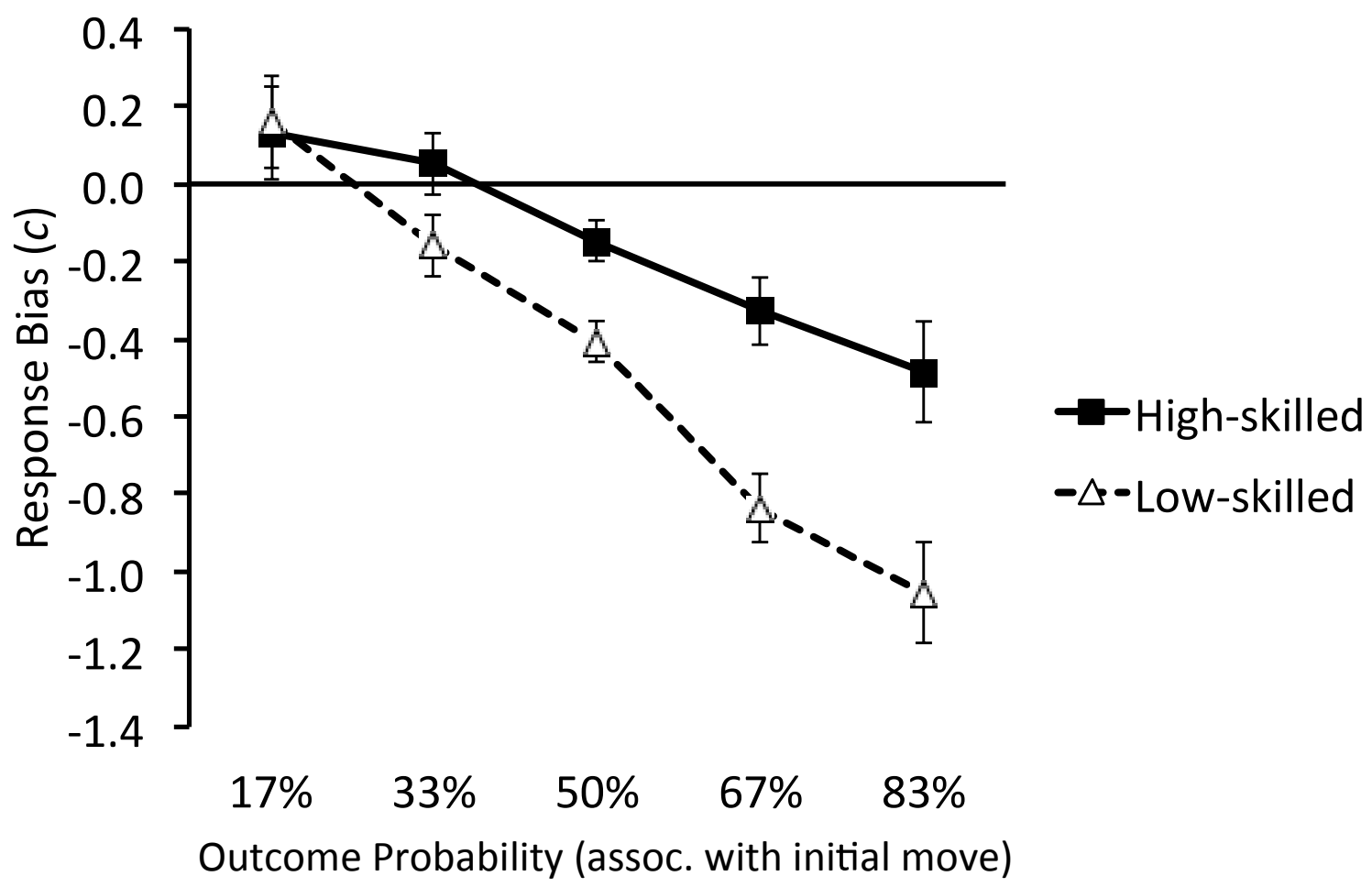

Figure 3. Bias toward judging actions to be genuine (-ve values) or deceptive (+ve values) for each outcome probability value. 


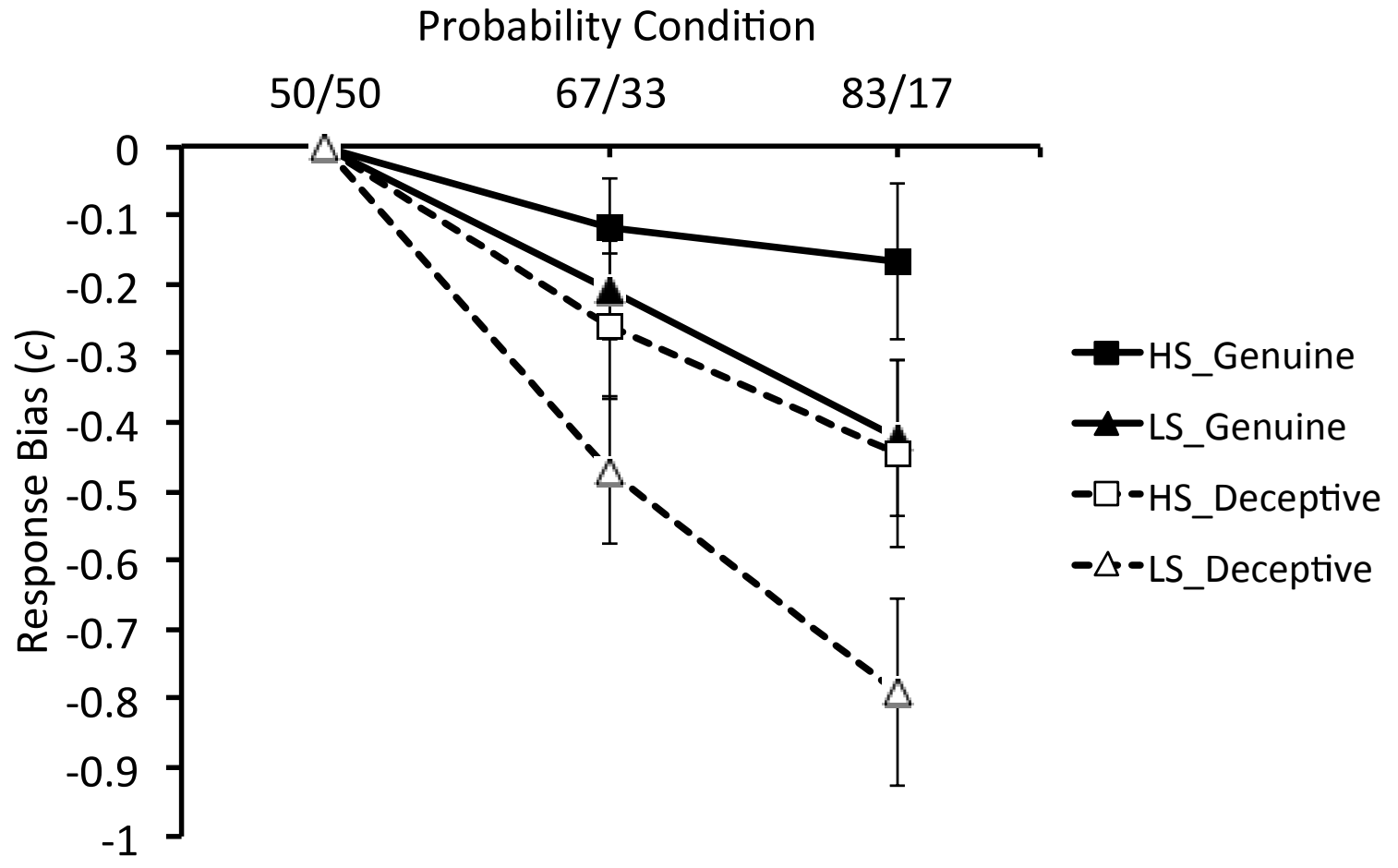

Figure 4. Bias toward responding congruent with the higher-probability value (-ve bias) on genuine and deceptive trials in each outcome probability condition. 


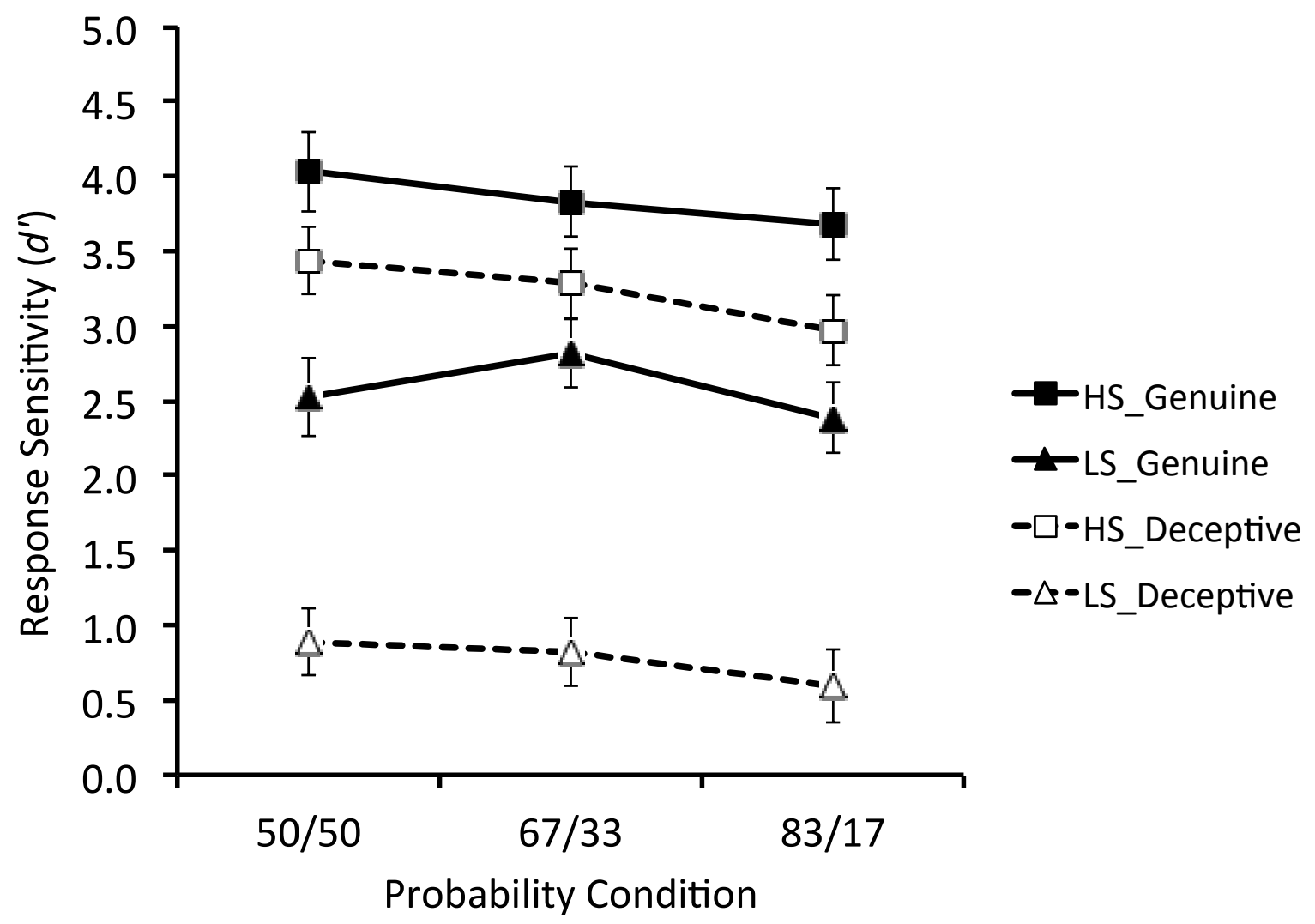

Figure 5. Sensitivity in discriminating between left and right action outcomes in the highskilled (HS) and low-skilled (LS) group when viewing genuine and deceptive trials in each outcome probability pairing. 


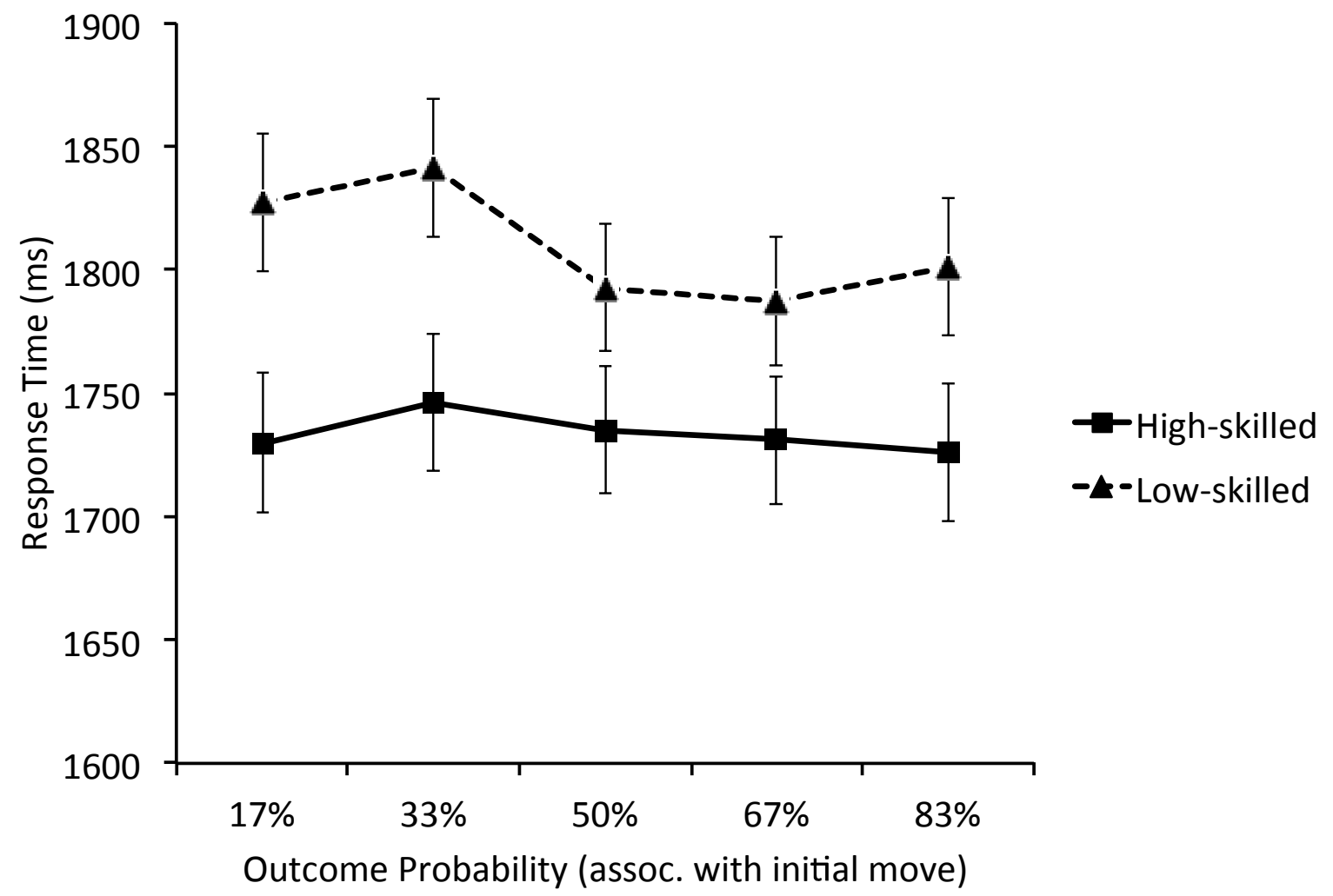

Figure 6. Mean response times for the high-skilled and low-skilled groups for each outcome probability. 
Knowledge is Power? Outcome Probability Information Impairs Detection of Deceptive Intent

Highlights

- We examined the effect of outcome probability information on detection of deception

- Probability information more strongly biased responses to deceptive actions

- Probability information more strongly biased responses of low-skilled players

- Knowledge of player tendencies impaired detection of deceptive intent 
Competing interests

There are no competing interests to declare. 